RPI-98-N116

FTUV 98/20

IFIC $98 / 20$

\title{
INCLUSIVE MUON CAPTURE IN LIGHT NUCLEI
}

\author{
Nimai C. Mukhopadhyay ${ }^{a, b}$, H. C. Chiang ${ }^{a, c}$, S. K. Singh ${ }^{a, d}$ and E. Oset ${ }^{a}$ \\ ${ }^{a}$ Departamento de Física Teórica and IFIC, Centro Mixto Universidad de Valencia- \\ CSIC, 46100 Burjassot (Valencia), Spain \\ ${ }^{b}$ Department of Physics, Applied Physics and Astronomy, Rensselaer Polytechnic In- \\ stitute, Troy, New York 12180-3590, USA \\ ${ }^{c}$ Institute of High Energy Physics, Academia Sinica, Beijing, P.R. China \\ ${ }^{d}$ Physics Department, Aligarh Muslim University, Aligarh, India 202002
}

\begin{abstract}
We study total muon capture rates in light $(\mathrm{A} \sim 6-18)$ nuclei, taking into account renormalizations of the nuclear vector and axial vector strengths. We estimate the influence in the results of uncertainties of the spin-isospin interaction parameter $g^{\prime}$ and nuclear densities. A few of these reactions are theoretical benchmarks for physics involving searches for neutrino oscillations. New experiments in muon capture in several targets are suggested, in the light of some discrepancies with theory, crudeness of some experimental results and relevance to neutrino physics.
\end{abstract}


Since its discovery in the forties [1], nuclear muon capture (NMC) has been a probe par excellence for both weak interaction studies and exploring nuclear dynamics [2]. In the context of QCD, the nucleus provides a new vacuum, wherein quark and gluon condensates can have entirely different values from those in the free hadrons [3], thereby opening the possibility of nuclear renormalization of the hadronic weak couplings $\llbracket 4$. On the other hand, particle-hole and/or isobar-hole excitations provide a more traditional theoretical setting for describing the nuclear renormalization dynamics [5]. We explore here the latter in NMC via the study of inclusive (or total) capture rates.

The importance of NMC in light nuclei (here, those with mass number $A<20$ ) transcends nuclear physics. Thus, muon capture reactions in ${ }^{12} \mathrm{C}$ and ${ }^{16} \mathrm{O}$, to take two examples, provide us with opportunities to test the nuclear dynamical models, useful in interpreting the neutrino reactions, for example, those studied by the KARMEN [6] and the LSND [7] collaborations. The latter have bearings on the question of the neutrino oscillations, thus, physics beyond the standard model. The inclusive NMC rates in these targets are important benchmarks for the theory of nuclear response, of great value in setting the scale of our understanding within the standard model. While inclusive NMC rates are generally difficult theoretically to compute [8, 9, 10, 11, 2], these are experimentally relatively easy to measure [12], particularly in muon factories, where high muon purity and a decent stopping rate are readily available [13. Regrettably, such experiments have been few and far between in the recent times [12]. Our theoretical work here hopes to elicit new experimental interest in this subject, of importance to nuclear physics and particle physics.

The reaction of our interest is

$$
\mu^{-}(1 S)+(A, Z) \rightarrow \nu_{\mu}+(A, Z-1)^{*},
$$

wherein the nucleus $(A, Z-1)^{*}$, formed in the reaction, is not experimentally detected. The rate $\Lambda_{c}$ of the inclusive reaction is experimentally inferred from the disappearance rate of the muon [2, 12].

Theoretically, three common ways of calculating $\Lambda_{c}$ are: (1) by computing and summing exclusive channels [10], (2) by using the sum rules [8] and (3) by using the elementary particle method [11]. We follow a different method [9], which assumes the reaction (1) proceeding as

$$
\mu^{-}(1 S)+[p] \rightarrow \nu_{\mu}+[n],
$$

where $[p]$ and $[n]$ are nucleons belonging to two different local Fermi seas, characterized by the Fermi momenta $k_{F, i}(r)(i=p, n)$, given by the medium densities $\rho_{i}(r)=$ $k_{F, i}^{3}(r) / 3 \pi^{2}$. Then, the differential rate for the reaction (2) is given, in the local density approximation, by [9]

$$
\frac{d \Lambda_{c}}{d E_{\nu}} \sim \sum \sum|T|^{2} \operatorname{Im} \bar{U}\left(q_{0}, \vec{q}\right)
$$

where we suppress in Eq. (3) phase space factors and measures of integration, shown fully in Ref. [9]; $|T|^{2}$ is the square modulus of the transition amplitude, suitably 
renormalized [9] in the nuclear medium, summed and averaged over the initial and final states respectively; the function $\bar{U}\left(q_{0}, \vec{q}\right)$ is the nuclear Lindhard function for the particle-hole excitation. The three-momentum transfer $\vec{q}$ is fixed by the outgoing neutrino momentum. In our approach the energy transfer, $q_{0}$, to the nucleus is given by

$$
q_{0}=E_{\mu}+Q^{\prime}-Q-E_{\nu} .
$$

Here $E_{\mu}$ is the available energy from the muon

$$
E_{\mu}=m_{\mu}-E_{\mu}^{1 S}
$$

where the second term on the right-hand side of (5a) is the muon binding energy. $Q^{\prime}$ comes into play in the nucleus with $N \neq Z$,

$$
Q^{\prime}=E_{n_{F}}-E_{p_{F}} ;
$$

$Q$ is the threshold for the nuclear reaction (1) to begin [14]

$$
Q=M_{f}-M_{i},
$$

the nuclear mass difference for the target and daughter nuclei. For light nuclei under consideration in this Letter (target nuclei between ${ }^{6} \mathrm{Li}$ and ${ }^{18} \mathrm{O}$ ), the $Q$ value varies from a low of $0.67 \mathrm{MeV}$, in the case of ${ }^{14} \mathrm{~N}^{\mu_{-}^{-}} \rightarrow{ }^{14} \mathrm{C}$ transitions, to a high of $21.15 \mathrm{MeV}$, in the case of the ${ }^{14} \mathrm{C}$ target, metastable, but still experimentally accessible, because of its very long life, leading to ${ }^{14} C \stackrel{\mu^{-}}{\rightarrow}{ }^{14} B$ transitions; indeed, $Q$ values exceeding $10 \mathrm{MeV}$ are quite common in light nuclei. We find a significant effect of the $Q$ value on the total capture rate in light nuclei, since the right-hand side of (3) is a sensitive function of the available energy in the reaction, (2).

The calculations in [10] use a RPA approach to nuclear structure. Kolbe et al. use a continuum RPA which sums up over the excited nuclear states above nucleon emission. Auerbach et al. use a standard RPA, including pairing, that allows one to include the contribution of all final states. In both cases the RPA evaluation reduces the results with respect to the calculation with the single particle orbitals, but the reduction is stronger in the calculations of Auerbach et al.

Our approach might look simplified with respect to the ones just mentioned, but in fact it is also an RPA approach built up from single particle states of an uncorrelated local Fermi sea. This method in practice is a very accurate tool when the excitation energy is sufficiently large such that relatively many excited states contribute to the process, and in particular if a large fraction of it comes from excitation to the continuum, as it is the case in $\mu^{-}$capture. The adaptation of the method to finite nuclei via the local density approximation has proved to be a rather precise technique to deal with inclusive photonuclear reactions [15], response functions in electron scattering [16] and deep inelastic scattering [17].

Obviously, because of its nature, the method only applies to inclusive processes, summing over relatively many final states and it is not meant to evaluate transitions to discrete states. 
The simplicity of the method, however, allows for improvements over the traditional methods: 1) the sum $\bar{\Sigma} \Sigma\left|T^{2}\right|$ is done relativistically; 2) the nucleon momentum of the Fermi sea is considered in the calculations which usually is taken equal to zero; 3$)$ the $Z_{\text {eff }}$ approximation is avoided and instead our evaluation uses directly the muon wave functions. Nonrelativistic muon wave functions are used, but the relativistic effects were evaluated in [9] and amounted to about $2 \%$. The $\mu^{-}$binding energy is also considered. 4) In addition, our approach builds up the RPA correlations by allowing the $\Delta h$ excitation on top of the $p h$ excitation considered in [10]. This is a major difference with the other theoretical RPA approaches mentioned [10]. A Landau-Migdal interaction is used to account for the propagation of these $p h$ and $\Delta h$ states in the nucleus.

The $\Delta h$ excitation leads to extra quenching of the transition strength with respect to the one found by Kolbe et al. The role of this $\Delta h$ excitation in the quenching of Gamow Teller transitions was emphasized by Rho and later by Brown and Rho in ref [4]. This quenching could be interpreted as an effective quenching of $g_{A}$, since this coupling term gives the largest contribution to the $\mu$ capture rate; we can, instead, talk in terms of quenching of the transition strength. The pseudoscalar current is also renormalized, but this term plays a smaller role in $\mu$ capture.

Our method was used in [9], where a good overall agreement with experiments over the periodic table was found. The method has proved even more accurate than anticipated in the ref. [9], when the improvements of the present paper are done. The basic framework of our approach has been described in detail in [9]. We stress here only significant improvements of this approach: (1) We use a new Lindhard function [18, which takes into account a shift of the neutron and proton Fermi seas due to the experimental $\mathrm{Q}$ value [14]. Its imaginary part coincides with the the results of the Eq.(3) in terms of the ordinary Lindhard function, but its real part is changed slightly with respect to the old one. The new Lindhard function avoids pathologies of the ordinary Lindhard function in the limit $\left(q_{0}, \vec{q}\right) \rightarrow 0$. This limit, however, does not occur in the muon capture kinematics, but it does play an important role in the neutrino scattering at low energies. (2) The experimental $Q$ value and the theoretical one, $Q^{\prime}$, are used in the evaluation as indicated in eqs. (3), (4). The Q-value in general is important in determining the NMC rate in light nuclei. (3) The uncertainties coming from the Landau-Migdal parameter $g^{\prime}$ are included by varying $g^{\prime}$ in the range [19]

$$
g^{\prime}=0.7 \pm 0.1
$$

This is an important parameter influencing the nuclear response in our approach. This parameter occurs in the particle-hole and the Delta-hole interactions [4, 50. (4) In many nuclei, the radial form of the nuclear density has parametric uncertainties. We take them also into account in our calculation by letting the density parameters vary within the experimental errors of [20].

In Table I, we display our calculated total capture rate $\Lambda_{c}$, obtained by integrating (3), for $g^{\prime}=0.7$ and for one set of standard nuclear density parameters [20]. The present approach has significantly improved the agreement between the theory and 
experiment, compared with results reported in [9].

The principal uncertainty of our theoretical estimate of the NMC rate stems from that of the parameter $g^{\prime}$. For the range of $g^{\prime}$, given in equation (6), this results in an uncertainty of about $\pm(5-7) \%$ in the NMC rate for all the nuclei considered in this paper and reported in table I. Thus, to take a physical example, that of NMC by ${ }^{12} C$, we vary $g^{\prime}$ from 0.6 to 0.8 and this results in a variation of the rate from $3.78 \times 10^{4} \mathrm{~s}^{-1}$ to $3.43 \times 10^{4} \mathrm{~s}^{-1}$, a $\pm 5 \%$ effect around the central value of $3.6 \times 10^{4} \mathrm{~s}^{-1}$, corresponding to $g^{\prime}=0.7$. Our experience with many electroweak and strong processes points towards a value of $g^{\prime}=0.7$ [15, 16, 17]. Similarly, other implicit parameters of the particle-hole or the Delta-hole interactions are determined from other processes.

The nuclear densities $\rho_{p, n}$, enter in our calculation via the local density approximation [9]. There are three cases, for A equal to 12, 14 and 16, in which radial uncertainties are readily investigated in our approach by calculating the capture rate for many sets of density parameters determined from electron scattering 20. They produce relatively small uncertainties of $\pm(2-3) \%$. The theoretical capture rates quoted for these nuclei in table 1 should be read with this additional uncertainty due to the nuclear densities. Thus, in ${ }^{12} C$, the total capture rate varies from $3.45 \times 10^{4} s^{-1}$ to $3.60 \times 10^{4} s^{-1}$, keeping the spin-isospin parameter fixed. This is a $\pm 2 \%$ variation due to uncertainty of the nuclear density around the central value. Assuming the uncertainties of $g^{\prime}$ and nuclear densities to be independent, we have a theoretical uncertainty in ${ }^{12} \mathrm{C}$ NMC rate of $\simeq 6 \%$ : this is a conservative estimate of our theoretical error. Thus, the ${ }^{12} C$ NMC rate is, according to our theoretical calculation,

$$
\Lambda_{c}\left({ }^{12} C\right)=(3.60 \pm 0.22) \times 10^{4} s^{-1} .
$$

This is in good agreement with the best experimental determination so far [12], taking the world average of the best determinations, with their errors in quadrature:

$$
\Lambda_{c}^{\exp }\left({ }^{12} C\right)=(3.80 \pm 0.10) \times 10^{4} s^{-1} .
$$

In the following, we compare our work with the recent work of Kolbe et al. and Auerbach et al. for this reaction.

Kolbe et al. [10 have done a continuum RPA calculation, which can compute with a reasonable accuracy the NMC rates to the particle-unbound states in ${ }^{12} B$. Using their calculation of the 1994 paper [10], we obtain the inclusive capture rates for the ${ }^{12} \mathrm{C}$ nucleus, wherein the particle bound states are not included for the excited nucleus (see their Table I). We get theoretical rates for two different potentials to be 3.42 and 3.34 in units of $10^{4} s^{-1}$. These numbers are slightly above the experimental value for the capture to the particle unbound states quoted by Kolbe et al. Taking a conservative lower limit of $7000 s^{-1}$ for the muon capture rate to the particle-bound states directly from the experiment, we can translate the calculation of Kolbe et al. as

$$
\Lambda_{c}\left({ }^{12} C\right) \simeq(4.04-4.12) \times 10^{4} s^{-1} .
$$

Auerbach et al. have several variations of their HF-RPA model and they obtain [10] 


$$
\Lambda_{c}\left({ }^{12} C\right) \simeq(3.09-3.64) \times 10^{4} s^{-1} .
$$

Recent researches in neutrino physics have brought $\mathrm{NMC}$ in ${ }^{12} C$ in sharper focus as a theoretical benchmark. One has to keep in mind the results of the recent $\left(\nu_{e}, e^{-}\right)$and $\left(\nu_{\mu}, \mu^{-}\right)$experiments [6, [], along with the results of some recent theoretical calculations 10, 21. The latter reaction is the inverse of the NMC. In particular, the recent LSND experiment has produced an inclusive $\left(\nu_{\mu}, \mu^{-}\right)$rate, which is in disagreement with the calculation of Kolbe et al. [10] by a factor of about 1.5. However, Auerbach et al. [10], in a recent paper find that this disagreement can be eliminated in their Hartree-Fock- Random-Phase Approximation (HF-RPA) calculation that includes pairing. With the present approach, we obtain a rate which is within $15 \%$ of the experimental result when the maximum uncertainties in the theoretical as well as experimental results are taken into account 21].

In the neutrino physics context, the NMC produces the following situation: while Kolbe et al. 10 obtain a result for NMC rate which is within $5 \%$ of the experimental result and explain the $\left(\nu_{e}, e^{-}\right)$inclusive cross sections rather well, they overestimate the inclusive $\left(\nu_{\mu}, \mu^{-}\right)$cross section by (45-50)\%. In the calculations of Auerbach et al. [10], it is possible in one version of their model to obtain a NMC rate $\left(\Lambda_{c}=\right.$ $\left.3.64 \times 10^{4} s^{-1}\right)$ in fair agreement with the experiment, but this version of the model predicts slightly higher values for the $\left(\nu_{\mu}, \mu^{-}\right)$inclusive cross sections and a large value for the $\left(\nu_{e}, e^{-}\right)$inclusive cross sections when compared with the experiments . On the other hand, the version of the model, which can explain the inclusive neutrino reactions , predicts a NMC rate of $3.09 \times 10^{4} s^{-1}$ which is smaller than the experimental value. Our present method, when applied to ${ }^{12} \mathrm{C}$ reproduces the experimental results of muon capture as well as those of the $\left(\nu_{e}, e^{-}\right)$inclusive cross sections quite well, but it also overestimates the experimental values for the $\left(\nu_{\mu}, \mu^{-}\right)$inclusive cross sections by about (15-20)\% [21]. This underscores the important role of the $N M C$ in selected targets as benchmark reactions to calibrate the nuclear theory, which is, in turn, used to delineate standard model physics. From this point of view, the NMC rates in ${ }^{12,13} \mathrm{C}$ and ${ }^{16} \mathrm{O}$ are particularly important.

The above discussion, comparing results of different accurate methods, gives us an idea of present theoretical uncertainties in calculations of weak nuclear reactions.

We now come to a very subtle physics in NMC, the isotope effect. In Table II, we display our calculations for the ratios of the rates of NMC for a pair of isotopes. We compare here our results with experimental ones wherever available, and the prediction of the well-known formula of Primakoff [8]:

$$
\Lambda_{c}\left(A^{\prime}, Z^{\prime}\right) / \Lambda_{c}(A, Z)=\frac{1-\delta\left(A^{\prime}-Z^{\prime}\right) / 2 A^{\prime}}{1-\delta(A-Z) / 2 A} .
$$

where $\delta \simeq 3.15$. The Primakoff formula yields Pauli blocking of the muon capture process rather approximately.

From Table II, we can see that our calculation does much better than the Primakoff formula in three out of four cases where there are data on isotope shifts. For example, our calculation yields for ${ }^{13} \mathrm{C} /{ }^{12} \mathrm{C}$ and ${ }^{18} \mathrm{O} /{ }^{16} \mathrm{O}$ ratios 0.82 and 0.78 , in fair agreement 
with the experimental values of $0.90 \pm 0.01$ and $0.86 \pm 0.15$ respectively, in contrast to the predictions of the Primakoff formula of 0.71 and 0.59 in the two cases. Our approach is able to take into account the special properties of individual target nuclei such as the shell closure in ${ }^{16} O$ and the large $Q$-value needed in ${ }^{14} C$. No such physics is included in the relatively simple formula (11).

Detailed calculations, such as those in [10], should likewise do better than the Primakoff formula. They also take into account the role of the experimental $Q$ values. The case of ${ }^{7} \mathrm{Li} /{ }^{6} \mathrm{Li}$ does not come up too good in our approach. This is understandable, since, in very light nuclei, the local density approximation can become less accurate.

We now make some remarks on the experimental situation and future prospects at the muon factories. It is important to have muon capture rates in light nuclei measured with high precision. In several targets, old [2] and newer [12 experiments disagree quite strongly (e.g. ${ }^{9} \mathrm{Be},{ }^{14} \mathrm{~N},{ }^{16} \mathrm{O}$ etc.), even though the more recent experiments are of higher statistical accuracy. While our theoretical estimates agree with most of the recent determinations of the $\Lambda_{c}$, there are significant disagreements in ${ }^{7} \mathrm{Li}$ and ${ }^{14} \mathrm{~N}$. Some targets, ${ }^{6} \mathrm{Li}$ and ${ }^{7} \mathrm{Li}$, have relatively poorly known $\Lambda_{c}$ and deserve better experimental determination. ${ }^{14} C$, being a radioactive nucleus, will pose experimental challenges to prepare a dense enough nuclear target. However, it is very interesting theoretically: the $Q$-value for $\mathrm{NMC}$ in this target is the largest in the nuclei studied here, with an enormous effect on the rate. Hopefully, the long half-life of ${ }^{14} \mathrm{C}$ would make an experiment with it feasible.

Our worst agreement with experiment comes in ${ }^{7} \mathrm{Li}$ and ${ }^{14} \mathrm{~N}$ targets. We predict the inclusive NMC rates $3.4 \times 10^{3}$ and $8.7 \times 10^{4} s^{-1}$ in these two cases, while the most accurate experiments give $(2.26 \pm 0.12) \times 10^{3} s^{-1}$ and $(6.93 \pm 0.008) \times 10^{4} s^{-1}$ respectively [12]. Given the quality of our agreement in other targets with experimental results, this disagreement invites an experimental reconfirmation, with a special care on the systematic errors of the experiments. Further experimental studies are also needed to investigate possible non-statisticality in the hyperfine states of the muonic atom [2], in particular, in the case of ${ }^{14} N$, due to hyperfine conversion.

In summary, we have studied total NMC rates in the framework of a theory wherein vector and axial-vector strengths in nuclei are appreciably renormalized due to particle-hole and Delta-hole correlations. Large effects from the consideration of the Q-values of the NMC are seen, in some nuclei. A reasonable $( \pm 0.1)$ variation of the Landau-Migdal parameter $g^{\prime}$ around its preferred value 0.7 translates into a $\pm(6-7) \%$ uncertainty of the NMC rate for the nuclei studied in this paper. Similarly the nuclear radial uncertainties in some of these nuclei contribute at most a $\pm(2-3) \%$ variation of the capture rate.

Since renormalizations of weak vector and axial-vector strengths, studied here, also occur in the electromagnetic and strong processes, we should interpret them as those for nuclear strengths rather than for weak nucleon couplings in nuclei. This has important consequences in our understanding of the nuclear QCD effects, in particular, the Bjorken sum rule in nuclei.

Precise theoretical understanding of the NMC in ${ }^{12} C,{ }^{13} \mathrm{C}$ and ${ }^{16} \mathrm{O}$ would provide benchmarks in low-energy neutrino physics, with important bearings on the issue of 
neutrino oscillations and physics beyond the standard model. Although small, there are still defferences between the predictions of different accurate models and it would be most desirable to examine and test these differences in precise experiments in future.

We thank Prof. J. Deutsch and Prof. D. Measday for valuable communications. Three of us (HCC, NCM and SKS) have the great pleasure of thanking Professor E. Oset for his warm hospitality at the Universidad de Valencia. One of us (NCM) is grateful for the generous support of "IBERDROLA de Ciencia y Tecnologia" and acknowledges the partial support of the U.S. Dept. of Energy. HCC and SKS acknowledge the support of the Ministerio de Educacion y Cultura of Spain in their sabbatical stays. This work is also supported in part by CICYT, Contract numbered AEN-96-1719.

Table I: Total muon capture rates in $s^{-1}$ in various target nuclei, compared with the most accurate experimental results cited in the literature, wherever available 12; A dash in the last column means that no data are available in that case; in case of two or more experimental results of comparable quality, we display the value with best precision here. We use $g^{\prime}=0.7$ and one standard set of radial densities 20].

$\begin{array}{llc}\text { Target } & \text { This calculation } & \text { Experiment } \\ { }^{6} \mathrm{Li} & 4.68 \times 10^{3} & (4.68 \pm 0.12) \times 10^{3} \\ { }^{7} \mathrm{Li} & 3.41 \times 10^{3} & (2.26 \pm 0.12) \times 10^{3} \\ { }^{9} \mathrm{Be} & 8.84 \times 10^{3} & (7.4 \pm 0.5) \times 10^{3} \\ { }^{10} \mathrm{~B} & 2.67 \times 10^{4} & (2.78 \pm 0.07) \times 10^{4} \\ { }^{11} \mathrm{~B} & 1.86 \times 10^{4} & (2.19 \pm 0.07) \times 10^{4} \\ { }^{12} \mathrm{C} & 3.60 \times 10^{4} & (3.76 \pm 0.04) \times 10^{4} \\ { }^{13} \mathrm{C} & 2.95 \times 10^{4} & (3.38 \pm 0.04) \times 10^{4} \\ { }^{14} \mathrm{C} & 2.34 \times 10^{4} & - \\ { }^{14} \mathrm{~N} & 8.67 \times 10^{4} & (6.93 \pm 0.008) \times 10^{4} \\ { }^{15} \mathrm{~N} & 6.34 \times 10^{4} & - \\ { }^{16} \mathrm{O} & 1.16 \times 10^{5} & (1.026 \pm 0.006) \times 10^{5} \\ { }^{17} \mathrm{O} & 1.06 \times 10^{5} & - \\ { }^{18} \mathrm{O} & 9.00 \times 10^{4} & (8.80 \pm 0.15) \times 10^{4}\end{array}$

Table II: Isotope effect (with $g^{\prime}=0.7$ ) calculated by us, compared with the Primakoff formula (Eq. (14)) and experiment 12]. 


$\begin{array}{llll}\text { Isotope pair } & \text { This calculation } & \text { Primakoff } & \text { Exp. } \\ { }^{7} \mathrm{Li} /{ }^{6} \mathrm{Li} & 0.73 & 0.47 & 0.48 \pm 0.03 \\ { }^{11} \mathrm{~B} /{ }^{10} \mathrm{~B} & 0.79 & 0.67 & 0.79 \pm 0.03 \\ { }^{13} \mathrm{C} /{ }^{12} \mathrm{C} & 0.82 & 0.71 & 0.90 \pm 0.01 \\ { }^{14} \mathrm{C} /{ }^{12} \mathrm{C} & 0.65 & 0.47 & - \\ { }^{14} \mathrm{C} /{ }^{13} \mathrm{C} & 0.79 & 0.66 & - \\ { }^{15} \mathrm{~N} /{ }^{14} \mathrm{~N} & 0.73 & 0.75 & - \\ { }^{17} \mathrm{O} /{ }^{16} \mathrm{O} & 0.91 & 0.78 & - \\ { }^{18} \mathrm{O} /{ }^{16} \mathrm{O} & 0.78 & 0.59 & 0.86 \pm 0.15 \\ { }^{18} \mathrm{O} /{ }^{17} \mathrm{O} & 0.85 & 0.75 & -\end{array}$




\section{References}

[1] M. Conversi, E. Pancini and O. Piccioni, Phys. Rev.68 (1945) 232.

[2] N. C. Mukhopadhyay, Phys. Rep. 30C (1977) 1; Nucl. Phys. A335 (1980) 111c; Invited Paper in Weak and Electromagnetic Interactions in Nuclei, P. Depommier, ed. Ed. Frontiers (Gif-sur-Yvette, 1990). T. W. Donnelly and R.Peccei, Phys. Rep. 50 (1979)1.

[3] See, for example, E. V. Shuryak, The QCD Vacuum, Hadrons and Superdense Matter, World Scientific (Teaneck, NJ), 1988. H. Riffert, H. Müther, H. Herold and H. Ruder, Matter at High Densities in Astrophysics, Springer (Berlin; New York, 1996), and refs. therein. M. C. Birse, hep-ph/9602266 (1996), and refs. therein.

[4] M. Ericson, Ann. Phys. (NY) 63 (1971) 562. M. Ericson, A. Figureau and C. Thevenet, Phys. Lett. 45B (1973) 19. M. Rho, Nucl. Phys.A231 (1974) 493. K. Ohta and M. Wakamatsu, Nucl. Phys. A234 (1974) 445. N. C. Mukhopadhyay, H. Toki and W. Weise, Phys. Lett. 84B (1979) 35. E. Oset and M. Rho, Phys. Rev. Lett. 42 (1979) 47;I. S. Towner and F. Khanna, Phys. Rev. Lett. 42 (1979) 51. G. E. Brown and M. Rho, Comm. Nucl. Part. Phys. 10 (1981) 201.

[5] E. Oset, P. Fernández de Córdoba, L. L. Salcedo and R. Brockman, Phys. Rep. 188 (1988) 80, and refs. therein.

[6] B. E. Bodmann et al. (KARMEN coll.), Phys. Lett. B332 (1994) 251.

[7] C. Athanassopoulos et al. (LSND coll.), nucl.-ex/9705001,9705002 (1997).

[8] A sample of these works include: H. Primakoff, Rev. Mod. Phys.31 (1959) 802; B. Goulard and H. Primakoff, Phys. Rev.135 (1964) B1139; J. Bernabéu and F. Cannata, Nucl. Phys. A215 (1973) 411. See N. C. Mukhopadhyay [2] and T. Suzuki et al. [12], for a fuller survey of these and other methods.

[9] H. C. Chiang, E. Oset and P. Fernández de Córdoba, Nucl. Phys. A510 (1990) 591. See also, S. K. Singh and E. Oset, Phys. Rev. C48 (1993) 1246. T. S. Kosmas and E. Oset, Phys. Rev. C53 (1996) 1409.

[10] E. Kolbe, K. Langanke and P. Vogel, Nucl. Phys. A613 (1997) 382. E. Kolbe, K. Langanke and P. Vogel, Phys. Rev. C50 (1994) 2576; N. Van Giai, N. Auerbach and A.Z. Mekjian, Phys. Rev. Lett. 46 (1981) 1444; N. Auerbach, N. Van Giai and O. K. Vorov, Phys. Rev. C56 (1997) R2368.

[11] C.W. Kim and S.L. Mintz, Phys. Rev. C31 (1985) 274; S.L. Mintz and M. Pourkaviani, Nucl. Phys. A594 (1995) 346.

[12] G. Bardin, J. Duclos, J. Joseph, A. Magnon, J. Martino and E. Zavattini, Phys. Lett.79B (1978) 52. J. Martino, Thèse d'Etat, Uni. Paris-Sud (1982). K. Ishida, 
J. H. Brewer, T. Matsuzaki, Y. Kuno, J. Imazaki and K. Nagamine, Phys. Lett.167B (1986) 31. T. Suzuki, D. F. Measday and J. P. Roalsvig, Phys. Rev. C35 (1987) 2212, and refs. therein.

[13] J. Deutsch and D. F. Measday, priv. comm. (1997).

[14] C. M. Lederer and V. S. Shirley, Table of Isotopes, Wiley (New York,1978).

[15] R.C. Carrasco and E. Oset, Nucl. Phys. A536 (1992) 445.

[16] A.Gil, PhD Thesis, University of Valencia 1996; A. Gil, J. Nieves and E. Oset, Nucl. Phys. A A627 (1997) 543.

[17] E. Marco, E. Oset and P. Fernández de Córdoba, Nucl. Phys. A611 (1996) 484; P. Fernández de Córdoba, E. Marco, H. Müther, E. Oset and A. Faessler, Nucl. Phys. A611 (1996) 514.

[18] E. Oset, D. Strottman, H. Toki and J. Navarro, Phys. Rev. C48 (1993) 2395

[19] G. E. Brown and W. Weise, Phys. Rep. C27 (1976) 1. H. Müther, Progr. Part. Nucl. Phys. 14 (1985) 123.

[20] C. W. de Jager, H. de Vries and C. de Vries, Atomic data and Nucl. Data Tables 14 (1974) 479.

[21] S. K. Singh, N. C. Mukhopadhyay and E. Oset, submitted to Phys. Rev. C. 1997 